\title{
The Construction of an Athlete's Individual Profile of Speed-Strength Preparedness
}

\author{
Pyanzin A.I. \\ Department of Theoretical Basis of Physical Education \\ I.Ya. Yakovlev State Pedagogical University Cheboksary, \\ Russia \\ pianzin@mail.ru
}

\author{
Pyanzina N.N. \\ Department of Physical Education and Sports \\ I.N. Ulyanov Chuvash State University, \\ Cheboksary, Russia \\ npianzina@mail.ru
}

\begin{abstract}
Achieving high results in any sport is impossible without compliance with the proportionality condition in the manifestation of motor abilities. The research problem is to determine the functional dependence of the results in free standing vertical jumps and weighted vertical jumps with different weights. Materials. Data analysis was carried out in 4 groups of subjects representing: powerlifting, kettlebell lifting, basketball, athletics. Were used: scientific literature analysis and summarizing, mathematical modeling; pedagogical testing; methods of mathematical statistics. The height of free standing vertical jumps and weighted vertical jumps with different weights from 11.5 to $110 \mathrm{~kg}$ on the shoulders was measured in the subjects. A total of 618 measurements were performed. Checking the adequacy of the equations used to calculate the height of weighted vertical jumps based on the height of free standing vertical jumps taking into account the masses of athletes' bodies and weights showed that it reflects the regularity of the jump height changes depending on the masses of the weights. The equation allows us to calculate individual model values of the jump height as the mass of the weights increases and to assess the proportionality in manifestation level of athlete's speed-strength abilities components. The revealed dynamics of jump height correspondence to the individual normative level allows us to judge insufficiently proportionate use of speed-strength abilities developing means within the framework of the physical training process in various sports, namely, insufficient attention to mainly high-speed and mainly strength components development.
\end{abstract}

Keywords—basketball; kettlebell lifting; athletics; powerlifting; speed-strength abilities; proportionality.

\section{INTRODUCTION}

Achieving high results in any sport is impossible without compliance with proportionality condition in motor abilities manifestation. This is also true for speed-strength abilities.

To assess athlete's physical fitness level model characteristics are used that describe quantitative guidelines in a number of informative motor tests. Model characteristics of athlete's physical fitness are necessary to compare the normative level of measured parameters with the real one. If there is a lag of the real level from the model in one or another test, it becomes necessary to introduce corrections to the content of the training program aimed at eliminating the identified lag.

As a rule, models construction is based on the data of a certain set of athletes. Since such models are applicable only to a certain group of athletes, it is necessary to create separate complexes of model characteristics for each sports discipline, qualification, sex and age.

In addition, the model level for a group is defined as the arithmetic mean of the test result, taking into account its spread. It follows that normative results of different motor tests that make up the model characteristics are not related to each other, and therefore, in principle, cannot take into account individual characteristics of a particular athlete.

This study discusses the ways to construct an individual model of athlete's speed-strength training. This approach is based on the fact that:

1) the man and the environment are two sides of a single system in which the environment acts as an external link of self-regulation and determines the structure, size and proportions, strength potential and features of body functioning;

2) high stability of environment characteristics, vitally important for the body, determines a rather narrow range of characteristics of its anatomical structure and physiological processes functioning, as well as body movements on support [11];

3) in motor actions aimed at solving different motor tasks performed using different types of supports (track, ice, water, mechanical device), having different duration, as well as repetitive or non-repetitive composition of motor elements, the body realizes the same biological potential;

4) the parameters of movements and final results in different exercises are interdependent, since they reflect the same level of individual biological potential of the organism. 
TABLE 1. QUANTITATIVE COMPOSITION OF TEST GROUPS

\begin{tabular}{|c|c|c|c|c|c|c|}
\hline 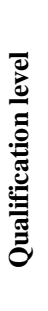 & 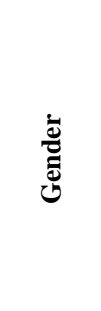 & : & 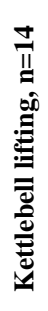 & 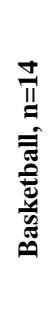 & 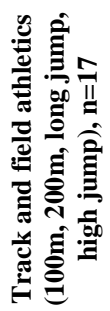 & 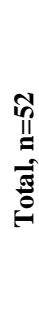 \\
\hline \multirow{2}{*}{$\stackrel{\Xi}{\stackrel{\Xi}{I}}$} & Male & & 3 & & & 3 \\
\hline & Female & 1 & 1 & & & 2 \\
\hline \multirow{2}{*}{$\begin{array}{l}\text { 包 } \\
\text { 王 }\end{array}$} & Male & 1 & 4 & 2 & 2 & 9 \\
\hline & Female & 1 & & & & 1 \\
\hline \multirow{2}{*}{ 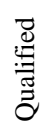 } & Male & 3 & 6 & 12 & 7 & 28 \\
\hline & Female & 1 & & & 8 & 9 \\
\hline
\end{tabular}

The free vertical jump is a generally accepted informative test for determining the speed-strength abilities level of an athlete. At the same time, a free vertical jump reflects only one of the sections of speed-strength abilities, namely the most rapid use of the strength potential of muscles that cause the body to accelerate upwards in the absence of any external resistance with the maximum manifestation of the speed and the minimum manifestation of the speed-strength abilities strength component.

The use of additional external weights increases the body weight, its inertness, slows its acceleration when the same individually specific muscle potential is manifested. So, with an increase in the mass of external weights, the height of jumping up should decrease proportionally, reflecting an increase in the proportion of strength component manifestations and a decrease in the speed component proportion of person's speed-strength abilities.

If we talk about free vertical jumps and weighted vertical jumps with different weights, the results of the same person should be interdependent and expressed by a certain function. Then, knowing the actual result of a free vertical jump or any weighted vertical jumps, it would be possible to calculate the entire chain of results in all other variants of this jump, and to construct an individual speed-strength profile on this basis. And to assess the level of speedstrength abilities not only in a single section when performing a free vertical jump, but also in all other sections in the range from the most "speed" to the most "strength" movements.

The determination of this functional dependence is the research problem. The solution of the problem would allow us to form an individual profile of speed-strength preparedness, calculating the quantitative values of the jump height with each weight according to the equation reflecting the relationship between them.

The object of the research are speed-strength abilities of qualified athletes.

The subject of the research is the structure of speedstrength preparedness of qualified athletes engaged in various sports.

The purpose of the study is to identify the functional dependence of the vertical jump height using different weights and to develop a test profile for assessment of speed-strength preparedness of qualified athletes engaged in various sports.

\section{MATERIALS AND METHODS}

The study used the following methods: analysis of scientific and methodical literature, mathematical modeling, pedagogical testing, methods of mathematical statistics.

Data analysis was carried out in 4 groups of subjects representing: powerlifting, kettlebell lifting, basketball, athletics (Table 1).

The height of free standing vertical jumps and vertical jumps with different weights from 11.5 to $110 \mathrm{~kg}$ on the shoulders was measured in the subjects. 3 attempts were recorded in each jump variant. A total of 618 measurements were performed. To conduct a comparative analysis, the maximum results in test exercises were used.

The height of jumping up was recorded with a measuring tape fixed on the support between the feet of the subject, which is a drum with a lock and a return mechanism. The upper end of the tape is attached to the subject's belt. Before performing the jump, the subject rises on his toes, the assistant fixes the initial value on the tape. Then, holding his hands behind his back (or on the neck of the rod), the subject crouches down and performs an upward jump from a place to a maximum height, pulling out a measuring tape. Landing should be performed on two legs in the take-off area, without displacement of the body in any horizontal direction. After landing, the assistant fixes the final value on the tape. The height of the jump is determined as the difference between the final and initial values of the measuring tape $\left(H=H_{\text {final }}-H_{\text {initial }}\right)$ in whole centimeters with rounding down.

\section{RESULTS AND DISCUSSION}

The starting point in this study is the view that the man and the environment act as two sides of a single system in which the environment is the external link of self-regulation, and the body is a material and functional reflection of the environmental conditions that determine its properties. Since the key environmental conditions (duration of the day, periodicity of day and night, the ratio of gases in the Earth's atmosphere, the gravity, the nature of the support) are invariant, they set certain limits in the manifestation of anatomical structure features and physiological processes functioning in the human organism, as well as the movements of the body on the support. 
The method of calculating the free standing vertical jump result is based on taking into account the gravitational coefficient which is the ratio of body mass with a weight on the shoulders to body mass without a weight. Figure 1 shows the logical basis of the algorithm for calculating the weighted vertical jump height.

The determination of take-off velocity relative value in an weighted vertical jump to the free standing vertical jump take-off velocity $\left(v_{r e l}\right)$ is based on the law of the body momentum conservation, according to which the momentum $(\mathrm{K})$ in an isolated material system remains unchanged [5]. That is, as the mass of the "athlete-weight" system increases, the take-off velocity proportionally decreases, so that their product continues to remain unchanged

Expressed in absolute values, humans' body weight is different due to differences in body mass, but with the same acceleration of gravity $\left(g=9.81 \mathrm{~m} / \mathrm{sec}^{2}\right)$. If the individual body mass and the gravity acceleration under the conditions of Earth's gravity are expressed by one, then the individual body weight, as their product, will also be equal to one:

This is a kind of starting point for further calculations.

A weighted vertical jump can be considered as a free standing vertical jump in conditions of increased gravity (above $1 \mathrm{~g}$ ), proportional to the total mass of the "athleteweight" system.

For example, an athlete weighing $70 \mathrm{~kg}(1 \mathrm{~m})$ performs a two-legged vertical jump with a $30 \mathrm{~kg}$ barbell on his shoulders $(0.43 \mathrm{~m})$. In this case, the mass of the "athleteweight" system will be more than one and will be $1.43 \mathrm{~m}$. With the Earth's acceleration of free fall, the equation will look like this:

or it can be written in a slightly different way:

Both equations are equivalent. They indicate that the body weight of a person with a barbell on his shoulders under standard Earth's gravity $(1.43 \mathrm{~m} \cdot 1 \mathrm{~g})$ is equal to the body weight of a person without a barbell on his shoulders under conditions of increased gravity $(1 \mathrm{~m} \cdot 1.43 \mathrm{~g})$. A variable, the value of which in this case is 1.43 units, can be denoted as the gravitational coefficient $\left(C_{g}\right)$ [8].

Hence, it is possible to calculate the height of a weighted vertical jump based on the result of a free standing vertical jump, taking into account the mass of the athlete and the weight. We took the free standing vertical jump as a point of reference for other jumping exercises because the body takeoff angle is directed perpendicular to the support and parallel to the gravity vector. The height of the jump is determined only by the ability of the muscles to give the body acceleration without additional movements (such as run-up or swings of the limbs). where:

$K$ - momentum, $\mathrm{kg} \cdot \mathrm{m} / \mathrm{sec}$

$m_{1}$ - athlete's body mass, $\mathrm{kg}$,

$v_{1}$ - free standing vertical jump take-off velocity, $\mathrm{m} / \mathrm{sec}$,

$C_{g}$ - gravity coefficient, rel. un.,

$v_{\text {rel }}$ - ratio of the weighted vertical jump take-off velocity to the free one, rel. un.

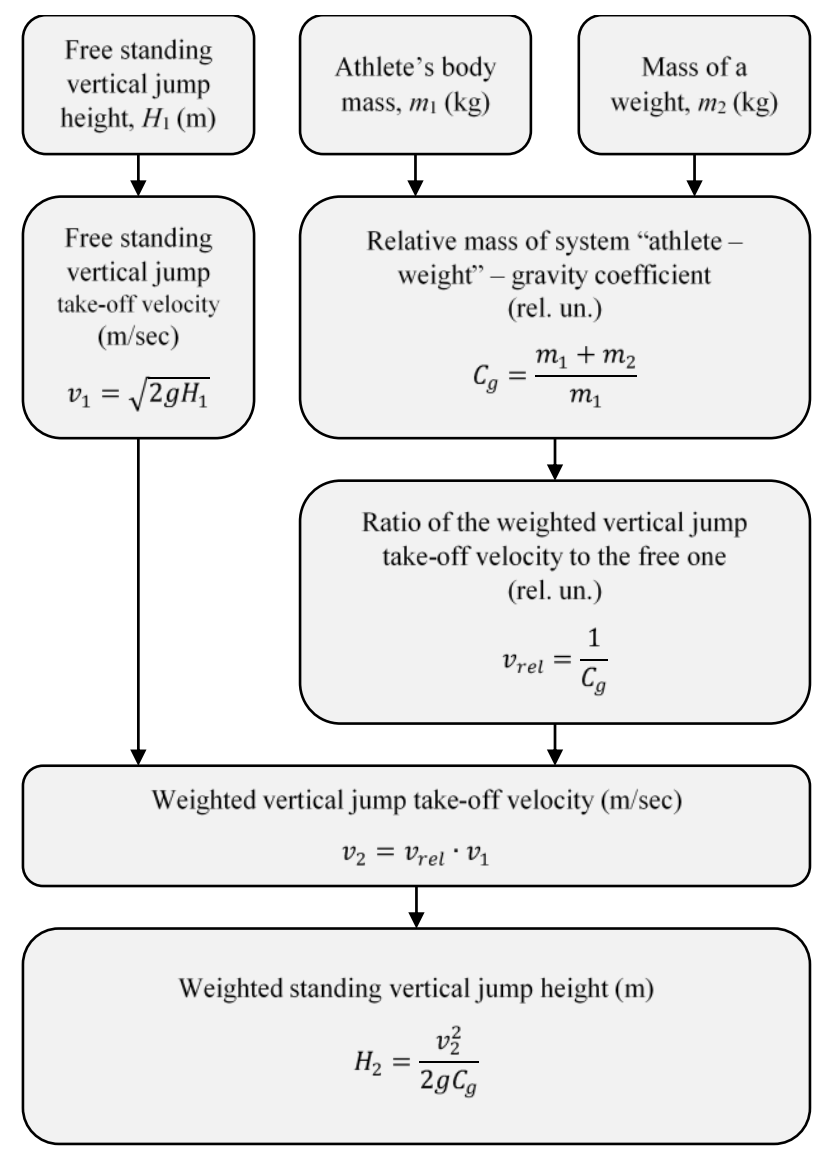

Fig. 1. Logic basis of algorithm for calculating the weighted standing vertical jump height 
Hence it follows that

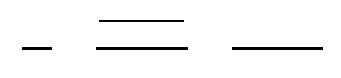

where:

$v_{r e l}$ - ratio of the weighted vertical jump take-off velocity to the free one, rel. un.

$C_{g}$ - gravity coefficient, rel. un.,

$m_{1}$ - athlete's body mass, $\mathrm{kg}$,

$m_{2}$ - mass of a weight, $\mathrm{kg}$.

We calculate the weighted standing vertical jump take-off velocity $\left(v_{2}\right)$ :

\section{$-\quad(7)$}

where:

$v_{1}$ - free standing vertical jump take-off velocity, $\mathrm{m} / \mathrm{sec}$,

$v_{2}$ - weighted vertical jump take-off velocity, $\mathrm{m} / \mathrm{sec}$,

$v_{\text {rel }}-$ ratio of the weighted vertical jump take-off velocity to the free one, rel. un.

$m_{1}-$ athlete's body mass, $\mathrm{kg}$;

$m_{2}$ - mass of a weight, $\mathrm{kg}$.

$g$ - gravity acceleration, $\mathrm{m} / \mathrm{sec}^{2}$,

$H_{1}$ - free standing vertical jump height, $\mathrm{m}$. $\left(H_{2}\right)$ :

We calculate the weighted standing vertical jump height

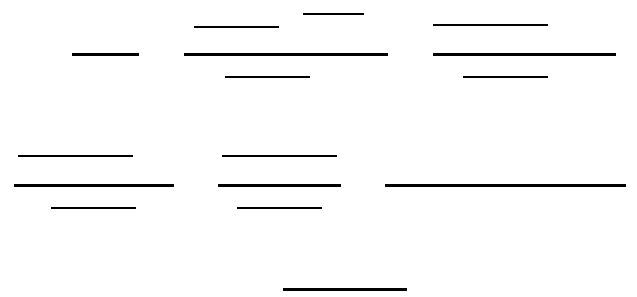

where:

$H_{1}$ - free standing vertical jump height, m;

$\mathrm{H}_{2}$ - weighted standing vertical jump height, m;

$m_{1}$ - athlete's body mass, $\mathrm{kg}$;

$m_{2}-$ mass of a weight, $\mathrm{kg}$.

The calculation of the jump height by the proposed equation (8) required checking of its adequacy degree and clarifying the variables. The check was carried out at comparison of estimated jump height with a real one at 52 qualified athletes representing 4 sports (Table 1).

If we equate the individual free standing vertical jump height to one, then as the mass of external weight on the shoulders increases, the height of jumping will proportionally decrease in accordance with the regularity described in the equation (8).

The comparison of real and calculated values of the jump height confirmed this pattern, but the results of the comparative analysis revealed some error when using the proposed equation. The real weighted standing vertical jump height was slightly higher than the calculated one.
These differences are not constant and tend to increase in proportion to the increase in weights. The general tendency in differences is described by the linear regression equation:

The introduction of the linear regression equation (9) into the equation for calculating of the jump height (8) as a correction factor made it possible to increase the calculating accuracy of the standing vertical jumps height with different weights, which can be used to form individual model characteristics of speed-strength preparedness, based on the individual result in a free standing vertical jump height (Fig. 2). The equation with the correction factor is presented below:

where:

$H_{1}$ - free standing vertical jump height, m;

$\mathrm{H}_{2}$ - weighted standing vertical jump height, m;

$m_{1}$ - athlete's body mass, $\mathrm{kg}$;

$m_{2}-$ mass of a weight, $\mathrm{kg}$.

The equation (10) helps not only to assess the overall speed-strength potential, but also to form an individual profile of speed-strength preparedness of an athlete, to reveal its structure, to determine the adequacy of speed or strength implementation on each of the profile "sections" of. It allows us to calculate the individual model values of the jump height as the weight increases, based on the result of free standing vertical jump, and to identify the proportionality in the level of individual components manifestation of speed-strength abilities of an athlete.

Thus, the lag of the real jump height from the model in the area of "heavy" weights may indicate a lack of strength component in the structure of speed-strength abilities manifestation. Lagging in the area of "light" weights (example in Fig. 3) can say about the lack of speed component in the structure of speed-strength abilities manifestation. The proportionate height ratio of jumps with different weights will be expressed in the speed-strength profile of an athlete in values close to the normative ones.

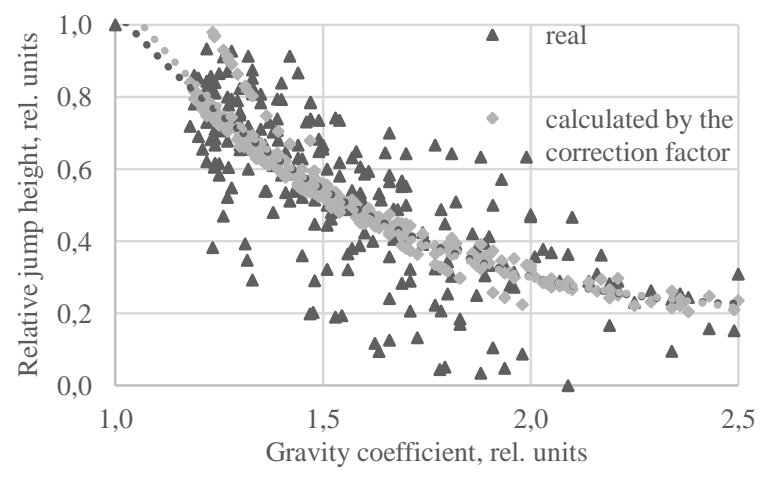

Fig. 2. Real and calculated relative standing vertical jump height by the correction factor 


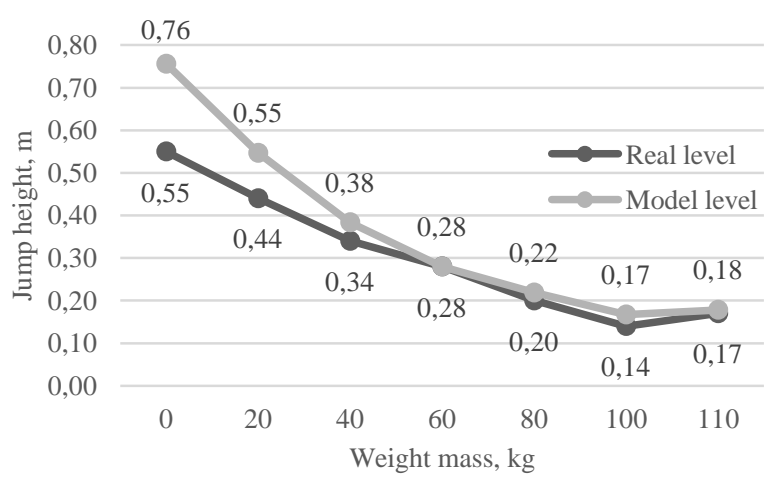

Fig. 3. Example of individual manifestation of speed-strength abilities in standing vertical jumps with different weight

Based on individual manifestations of athlete's performance, the coach has an effective tool for identifying the reserve of the jump height at any point of the speedstrength profile, which, in turn, will help to highlight the most relevant direction of training load in terms of speedstrength abilities development.

- Taking into account high calculation accuracy of individual weighted standing vertical jump height and sufficient volume of saved-up empirical data in various sports, we checked how the structure of speed-strength abilities manifestation in qualified athletes changes.

Tenfold smoothing of dynamic series based on the moving average method at three points was carried out to identify the trend in the jump height change as the mass of the weight increased [1]. Smoothed dynamics of relative jump height correspondence (Fig. 4) to individually regulatory level allows us to see the sections of the speedstrength profile, to a greater or lesser extent corresponding to normative results depending on the mass of a weight.
Smaller quantitative values of the gravitational coefficient characterize the predominant manifestation of the speed, and larger ones characterize the strength component in the structure of speed-strength abilities, forming the scale "speed-strength".

The graph in figure 4 shows the smallest lag of the jump height from the individual normative level in the range of weights from 1.35 to 1.65 rel. un.

In the range of weights $1.00-1.35$ rel. un. there is a more noticeable lag of real results from the individual normative level of an athlete. In our opinion, this lag shows common for sports shortcomings in the development of mainly highspeed component in development of speed-strength abilities.

Excess weights level of 1.65 rel. un. in athletes is accompanied by gradual lag of free standing vertical jump height from individually normative level and unstable dynamics of this indicator that can refer to available shortcomings of mainly strength component in development of speed-strength abilities.

Based on the data obtained, it is possible to judge indirectly the insufficiently proportionate use of speedstrength abilities developing means within the process of physical training in various sports, namely, insufficient attention to the development of mainly speed and mainly strength components of these abilities.

\section{CONCLUSION}

Thus, the adequacy of equations used to calculate the weighted standing vertical jump height based on free standing vertical jump height taking into account the masses of athletes' bodies and weights showed that it reflects the pattern of changes of the jump height depending on the masses of weights. The introduction of the correction factor in the proposed equation will help to provide a more

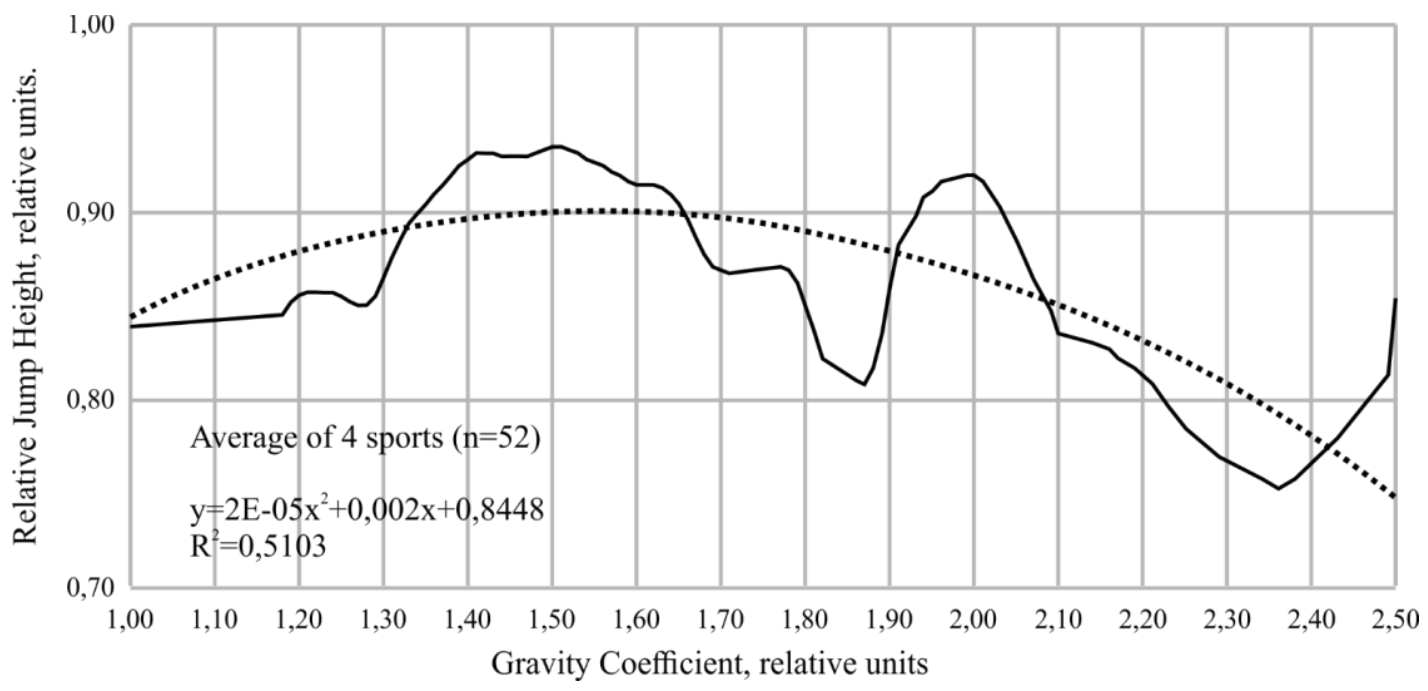

Fig. 4. Smoothed dynamics of compliance with relative jump height to individual normative level in test groups 
trained male athletes. Journal of Strength and Conditioning Research. 2012, 26, pp. 63-72.

[16] Woolstenhulme M.T., Griffiths C.M., Woolstenhulme E.M., Parcell A.C. Ballistic stretching increases flexibility and acute vertical jump height when combined with basketball activity. Journal of Strength and Conditioning Research. 2006, 20, pp. 799-803.

[1] Balandin V.I., Bludov Yu.M., Plahtienko V.A. Forecasting in sport. Moscow: Physical Education and Sport. 1986, pp. 91-99.

[2] Beckett J.R., Schneiker K.T., Wallman KE., Dawson B.T., Guelfi K.J. Effects of static stretching on repeated sprint and change of direction performance. Medicine and Science in sports and Exercise. 2009, 41, pp. 444-450.

[3] Chaouachi A., Castagna C., Chtara M., Brughelli M., Turki O., Galy O., et al. Effect of warm-ups involving static or dynamic stretching on agility, and jumping performance in trained individuals. Journal of Strength and Conditioning Research. 2010, 24, pp. 2001-2011.

[4] Curry B.S., Chengkalath D., Crouch G.J., Romance M., Mans P.J. Acute effects of dynamic stretching, static stretching, and light aerobic activity on muscular performance in women. Journal of Strength and Conditioning Research. 2009, 23, pp. 1811-1819.

[5] Donskoy D.D. Saving and changing the amount of movement of the system.

[6] Biomechanics with the basics of sports technique]. Moscow: Physical Education and Sport. 1971, pp. 185-187.

[7] Fletcher I.M. The effect of different dynamic stretch velocities on jump performance. European Journal Applied Physiology. 2010, 109, pp. 491-498.

[8] Fletcher I.M., Anness R. The acute effects of combined static and dynamic stretch protocols on fifty-meter sprint performance in trackand-field athletes. Journal of Strength and Conditioning Research. 2007, 21, pp. 784-787.

[9] Pyanzin A.I. Calculation of the result in a vertical jump with weight based on the result in a vertical jump without weight. Chuvash State Pedagogical University named after I.Y. Yakovlev Bulletin. 2017, 4(96), pp. 175-182.

[10] Pyanzin A.I. Sport preparation of jumpers. Theory and practice of physical culture. 2004, 370.

[11] Pyanzin A.I. The proportionality of the parameters of the interaction of the body with the support when running and jumping. Science and sport: current trends. 2016, 3(12), pp. 41-45.

[12] Pyanzin A.I. The structure of speed-strength readiness of the qualified athletes, going in for different kinds of sport. Russian Journal of Physical Education and Sport. 2019, 14(1), pp. 70-76.

[13] Pyanzin A.I., Pyanzina N.N. Criteria for model characteristics of athletes' special physical readiness creation. Physical culture and sports in high school: modern tendencies and practices: materials of All-Russian scientific-practical conference. Stavropol: SKFU. 2015, pp. 172-176.

[14] Pyanzin A.I., Pyanzina N.N. Kinematics of motor actions as the basis of model characteristics of athletes' special physical readiness formation. Innovative technologies in training athletes in wrestling: materials of the I All-Russian scientificpractical conference. Naberezhnye Chelny: Naberezhnye Chelny Branch of Povolzhskaya State Academy of Physical Culture, Sport and Tourism. 2014, pp. 207-210.

[15] Turki O., Chaouachi A., Behm D.G., Chtara H., Chtara M., Bishop D., et al. The effect of warm-ups incorporating different volumes of dynamic stretching on 10 - and 20-m sprint performance in highly 